\title{
Altered time structure of neuro-endocrine-immune system function in lung cancer patients
}

\author{
Gianluigi Mazzoccoli*1 , Gianluigi Vendemiale2, Angelo De Cata'1, Stefano Carughi1 and Roberto Tarquini3
}

\begin{abstract}
Background: The onset and the development of neoplastic disease may be influenced by many physiological, biological and immunological factors. The nervous, endocrine and immune system might act as an integrated unit to mantain body defense against this pathological process and reciprocal influences have been evidenced among hypothalamus, pituitary, thyroid, adrenal, pineal gland and immune system. In this study we evaluated differences among healthy subjects and subjects suffering from lung cancer in the 24-hour secretory profile of melatonin, cortisol, TRH, TSH, FT4, GH, IGF-1 and IL-2 and circadian variations of lymphocyte subpopulations.

Methods: In ten healthy male volunteers (age range 45-66) and ten male patients with untreated non small cell lung cancer (age range 46-65) we measured melatonin, cortisol, TRH, TSH, FT4, GH, IGF-1 and IL-2 serum levels and percentages of lymphocyte subpopulations on blood samples collected every four hours for 24 hours. One-way ANOVA between the timepoints for each variable and each group was performed to look for a time-effect, the presence of circadian rhythmicity was evaluated, MESOR, amplitude and acrophase values, mean diurnal levels and mean nocturnal levels were compared.

Results: A clear circadian rhythm was validated in the control group for hormone serum level and for lymphocyte subsets variation. Melatonin, TRH, TSH, GH, CD3, CD4, HLA-DR, CD20 and CD25 expressing cells presented circadian rhythmicity with acrophase during the night. Cortisol, CD8, CD8bright, CD8 dim, CD16, TCR 1 and $\delta T C S 1$ presented circadian rhythmicity with acrophase in the morning/at noon. FT4, IGF-1 and IL-2 variation did not show circadian rhythmicity. In lung cancer patients cortisol, TRH, TSH and GH serum level and all the lymphocyte subsubsets variation (except for CD4) showed loss of circadian rhythmicity. MESOR of cortisol, TRH, GH, IL-2 and CD16 was increased, whereas MESOR of TSH, IGF-1, CD8, CD8bright, TcR $\delta 1$ and $\delta$ TCS1 was decreased in cancer patients. The melatonin/ cortisol mean nocturnal level ratio was decreased in cancer patients.

Conclusion: The altered secretion and loss of circadian rhythmicity of many studied factors observed in the subjects suffering from neoplastic disease may be expression of gradual alteration of the integrated function of the neuroimmune-endocrine system
\end{abstract}

\section{Background}

There are close relations between endocrine, nervous and immune system. Cortisol has a well recognized influence on immune function, inducing significant immunosuppression, characterized by the reduced cellular and humoral response of monocytes and $\mathrm{B}$ and $\mathrm{T}$ lympho-

\footnotetext{
*Correspondence: g.mazzoccoli@tin.it

1 Unit of Internal Medicine and Chronobiology Unit, IRCCS Scientific Institute and Regional General Hospital, "Casa Sollievo della Sofferenza", Opera di Padre Pio da Pietrelcina S.Giovanni Rotondo (FG), Italy

Full list of author information is available at the end of the article
}

cytes [1]. Melatonin, hormone secreted by the pineal gland, is able to influence the secretion of many endocrine glands, modulates the function of the immune system, shows antioxidative and oncostatic property and its production is under the control of the nervous system [25]. The pineal hormone directly stimulates activated helper $\mathrm{T}$ lymphocytes and plays an immunomodulatory role by opioid peptides [6] and by a thyroid-independent influence on the thymic function mediated by thyrotropin-releasing hormone (TRH) and thyroid-stimulating 
hormone (TSH) [7]. The insulin like growth factor (IGF)1 is a potent mitogen for many cancer cell lines and the stimulation of IGF-1 receptor is necessary for proliferation of many cells in vivo and in vitro, often in conjunction with other growth factors [8]. Besides, recent studies have put in evidence that growth hormone $(\mathrm{GH})$ and IGF-1 have an important role in stimulating lymphocyte production and function [9]. Abnormalities in hormone serum levels and in the proportions of various lymphocyte subpopulations have been found in a number of tumors [10]. The purpose of this study was to evaluate the 24-hour profiles of lymphocyte subsets and neuroendocrine hormones with the aim to highlight alterations in patients suffering from lung cancer.

\section{Methods}

The study was approved by the local Scientific and Ethical Committee (approval number MGRL001 approval date 6/4/2008). After obtainment of informed consent peripheral blood samples were collected at intervals of four hours for 24 hours starting at 06:00 h from ten healthy subjects (HS, mean age \pm s.e. $57.2 \pm 2.7$, range 45 66) and from ten patients with untreated non small cell lung cancer (CP, mean age \pm s.e. $56.6 \pm 0.2$, range 46-65). Inclusion criteria for healthy subjects were sex (male), age ( $<80$ years), BMI ( $>25$ and $<30)$, normal physical activity level, no psychiatric disorder, no alcohol intake, no chronic conditions, normal blood pressure level. In all control subjects healthy status was assessed by medical history and physical examination, basal screening blood and urine test, ECG, chest $\mathrm{X}$ ray, upper and lower abdominal ultrasound scan. Inclusion criteria for subjects suffering from lung cancer were sex (male), age ( $<80$ years), BMI ( $>25$ and $<30$ ), normal physical activity level, performance status $>80 \%$ by Karnofsky performance status scale or $<2$ by ECOG score, no psychiatric disorder, no alcohol intake, no chronic conditions, normal blood pressure level, tumor cell type (non small cell lung cancer, NSCLC) and the extent of the tumor was evaluated by clinical examination, bronchoscopy, computed tomography (CT) of the brain, chest, upper abdomen and ultrasonography of the liver. There were 3 cases of squamous cell carcinoma and 7 of adenocarcinoma. The pathological diagnosis was based on light microscopy according to the WHO classification. Tumors were staged according to the TNM classification of the International Union Against Cancer staging system after reviewing the clini$\mathrm{cal}$, radiologic, and pathologic data. The numbers of pT1, pT2, and pT3-4 cases were 3,5 , and 2 respectively. All 10 cases showed metastasis to regional lymph nodes. Regarding stage, the numbers of stage I, II, and III cases were 2,5 , and 3 respectively. The groups were matched closely to avoid sex, body mass index and seasonality of sampling related bias and all subjects were submitted to the same social routine, with identical mealtimes and sleep/wakefulness rhythm in the week preceding the sampling (lights on at 07:00 a.m. and lights out at 10:00 p.m., thereby supplying the subjects with 15 hours of light and 9 hours of darkness per day, 15:9 L:D). During the overnight sampling period a dim blue light $(<100$ lux $)$ was used. In each blood sample we measured melatonin, cortisol, TRH, TSH, free thyroxine, GH, IGF-1 and IL-2. Blood was centrifuged immediately after collection and frozen at $-20^{\circ} \mathrm{C}$ for later determination. All samples were analyzed in duplicate in a single assay; the intrassay and interassay coefficients of variation were below $13 \%$ and $16 \%$ respectively for melatonin, $10 \%$ and $9 \%$ for cortisol, $5 \%$ and $6 \%$ for TRH, $8 \%$ and $7 \%$ for TSH, $4 \%$ and $6 \%$ for $\mathrm{FT}_{4}, 5 \%$ and $3 \%$ for $\mathrm{GH}, 3 \%$ and $8 \%$ for IGF-1, $5 \%$ and $7 \%$ for IL-2. Standard curves were run with every assay and the experimental values were derived from the curves. We measured melatonin by radioimmunoassay (Melatonin Radioimmunoassay Kit, Nichols Institute Diagnostics), cortisol by polarized light immuno-fluorescence assay (Cortisol TDx/TDxFLx, Abbott Laboratories), TRH by radioimmunoassay ("Frederic Joliot-Curie" National Research Institute for Radiobiology and Radiohygiene, Budapest, Hungary), TSH by immunoenzymatic assay (Enzymun-Test TSH, Boehringer Mannheim Immunodiagnostics), $\mathrm{FT}_{4}$ by immunoenzymatic assay (EnzymunTest $\mathrm{FT}_{4}$, Boehringer Mannheim Immunodiagnostics), GH by immunoenzymometric assay (AIA-PACK HGH, Tosoh, Japan) and IGF-1 by radioisotopic assay (IGF-1 $100 \mathrm{~T}$ Kit, Nichols Institute Diagnostics), IL-2 by immunoenzymatic assay (IL-2 EIA, Technogenetics). In each blood sample we analyzed lymphocyte subpopulations (CD3, CD4, CD8, CD8 bright, CD8 dim, CD16, CD20, CD25, HLA-DR, TcR $\delta 1, \delta$ TcS1) on peripheral blood anticoagulated with sodium ethylenediamine tetraacetic acid (EDTA). Analyses of lymphocyte subpopulations were performed on unfixed cell preparations with a fluorescence activated cell sorter (FACScan, Becton-Dickinson FACS Systems, Sunnyvale, California) and a panel of monoclonal antibodies (mAbs) to lymphocyte surface antigens (Ortho Diagnostic Systems: OKT3, OKT4, OKT8, OK-NK, OKB20, OKT26a, OK-DR; Thermo-Scientific, Rockford, IL, USA: TcR $\delta 1$ and $\delta$ TcS1). mAbs were directly conjugated with phycoerythrin (PE) and to fluorescein isothiocyanate (FITC). $10 \mu \mathrm{lmAbs}$ were added to $100 \mathrm{ml}$ EDTA blood in Trucount tubes (BD Biosciences, San Jose, CA). After a 15-min incubation at room temperature the erythrocytes were disintegrated and after centrifugation the supernatants were washed with PBS. Non-lymphocytic cells contaminating the preparations were excluded from analysis using scatter gates set on the $90^{\circ}$ light scatter profile. At least 10000 cells were acquired on the FACScan. Absolute counts of T cell subsets were 
calculated based on the proportion of the respective $\mathrm{T}$ cell subpopulation and on absolute counts obtained by the procedure. The number of fluorescent cells was expressed as a percentage of the total lymphocytes.

\section{Statistical analysis}

Statistical evaluation of hormone serum levels and lymphocyte subpopulation values was performed by noninferential descriptive biometric analyses, including oneway ANOVA performed between the timepoints for each variable and each group on original data and on data transformed as \% of their individual $24 \mathrm{~h}$ mean to look for a time-effect, Student's $t$ test and Mann-Whitney rank sum test, where appropriate, on MESOR, amplitude and acrophase values and on mean diurnal and nocturnal levels; a $p$ value $\leq 0.05$ was considered significant. The data were also analyzed by an inferential temporal descriptive biometric analysis using the methods named Single Cosinor and Population Mean Cosinor, based on a leastsquares fit of a cosine curve to individual and grouped time series data, testing the occurrence of a $24 \mathrm{~h}$ rhythm (whether the zero-amplitude assumption is rejected at a probability level $p \leq 0.05$ ) and quantifying the parameters MESOR, Amplitude and Acrophase of the rhythm. MESOR is the acronym for Midline Estimating Statistic of Rhythm and defines the rhythm-determined average. Amplitude is the measure of one half the extent of rhythmic change in a cycle estimated by the function used to approximate the rhythm. Acrophase, measure of timing, is the phase angle of the crest time in the function appropriately approximating a rhythm, in relation to the specified reference timepoint. Chronobiologic analysis was performed with Cosinor 2.2. ANOVA, Student's $t$ test and Mann-Whitney rank sum test were performed with SigmaPlot11.0 [11].

\section{Results}

In the healthy subjects ANOVA showed a time effect for cortisol, melatonin, TRH, TSH, GH, CD3, CD4, CD8, CD8 $8^{\text {bright }}, \mathrm{CD} 8^{\mathrm{dim}}, \mathrm{CD} 16, \mathrm{TcR} \delta 1$ and $\delta \mathrm{TcS} 1$ and a clear circadian rhythm was validated for the variation of hormone serum levels and of lymphocyte subset percentages: melatonin, TRH, TSH, GH, CD3 (total T cells), CD4 ( $T$ helper/inducer subset), HLA-DR (B cells and activated T cells), CD20 (total B cells) and CD25 (T activated lymphocytes with expression of the $\alpha$ chain of IL-2 receptor) presented circadian rhythmicity with nocturnal acrophase. Cortisol, CD8 (T suppressor/cytotoxic subset), CD8bright ( $\mathrm{T}$ suppressor subset), CD8 $\operatorname{dim}$ ( T cytotoxic subset), CD16 (natural killer cells), TcR $\delta 1$ (epitope of the constant domain of $\delta$ chain of TCR) and $\delta$ TcS1 (epitope of the variable domain of $\delta$ chain of TCR) presented circadian rhythmicity with diurnal acrophase. FT4, IGF-1 and IL-2 variation did not show circadian rhythmicity. In lung cancer patients ANOVA showed a time effect for cortisol, melatonin, TSH, CD4, CD16, CD25, TcR 81 and IL2. Cortisol, TRH, TSH and GH serum level and all the lymphocyte subsubsets variation (except for CD4) showed loss of circadian rhythmicity. MESOR of cortisol, TRH, GH, IL-2 and CD16 was increased in cancer patients, whereas MESOR of TSH, IGF-1, CD8, CD8 ${ }^{\text {bright, }}$ $\mathrm{TcR} \delta 1$ and $\delta \mathrm{TcS} 1$ was decreased in cancer patients (Figure 1 , figure 2 and figure 3 ).

When we compared mean diurnal levels (mean of 06:00h-10:00h-14:00h) GH (p < 0.001) and CD16 (p < $0.01)$ levels were higher in cancer patients, whereas IGF1 $(\mathrm{p}<0.001), C D 8(\mathrm{p}=0.03)$ and CD8 ${ }^{\text {bright }}(\mathrm{p}=0.001)$ levels were lower in cancer patients. When we compared mean nocturnal levels (mean of 18:00h-22:00h-02:00h) cortisol $(\mathrm{p}=0.02)$, TRH $(\mathrm{p}=0.03), \mathrm{GH}(\mathrm{p}<0.01)$, CD16 $(\mathrm{p}<$ $0.0001)$ and CD25 ( $\mathrm{p}=0.03)$ levels were higher in cancer patients, whereas melatonin $(\mathrm{p}=0.04)$, TSH $(\mathrm{p}=0.02)$, IGF-1 ( $\mathrm{p}<0.0001)$, CD8 ( $\mathrm{p}=0.01), \mathrm{CD}^{\text {bright }}(\mathrm{p}=0.001)$, CD20 ( $\mathrm{p}=0.03), \operatorname{TcR} \delta 1(\mathrm{p}=0.001)$ and $\delta \mathrm{TcS} 1(\mathrm{p}=0.02)$ levels were lower in cancer patients. The melatonin/cortisol mean nocturnal level ratio was decreased in cancer patients $(p=0.01)$

Table 1 shows chronobiological data derived from best fitting sine curves (fitted period:24 hours $=360^{\circ}$ ), F and $\mathrm{p}$ value from ANOVA performed between the timepoints for each variable and $\mathrm{p}$ value from Student's t test and Mann-Whitney rank sum test, where appropriate, on MESOR, amplitude and acrophase values between healthy subjects and lung cancer patients. Figure 1 and 2 show $x-y$ plot depicting 24-hour time qualified profiles of lymphocyte subset percentages and hormone serum levels.

\section{Discussion and Conclusion}

Body homeostasis is maintained by a perfect integration among the nervous, endocrine and immune system involving different glands and organs and guided by a spatio-temporal sequence of tissue-specific events, hormone and cyto-chemokine/receptor interaction and modulation processes. The pineal gland is innervated by post-ganglionic nervous fibers coming from the superior cervical ganglion, that receives fibers from the suprachiasmatic nucleus of hypotalamus, innervated by the retinohypothalamic tract. Changes in lighting condition influence activity of the retino-hypothalamic-pineal system, with inhibiting effect on melatonin production, controlled also by an endogenous free-running pacemaker located in the suprachiasmatic nucleus [12]. The melatonin mediated photoperiodic message is a fundamental cue for the circadian and seasonal coordination of biological functions. Melatonin plays a role of immunomodulation by opiatergic ways and stimulates activated helper $\mathrm{T}$ lymphocytes to produce opioid agonists and cytokines 

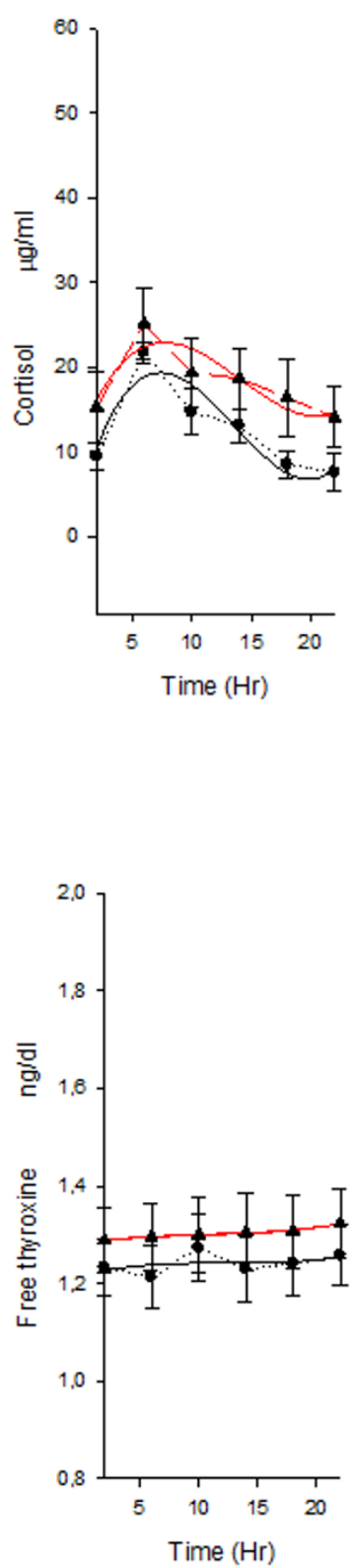
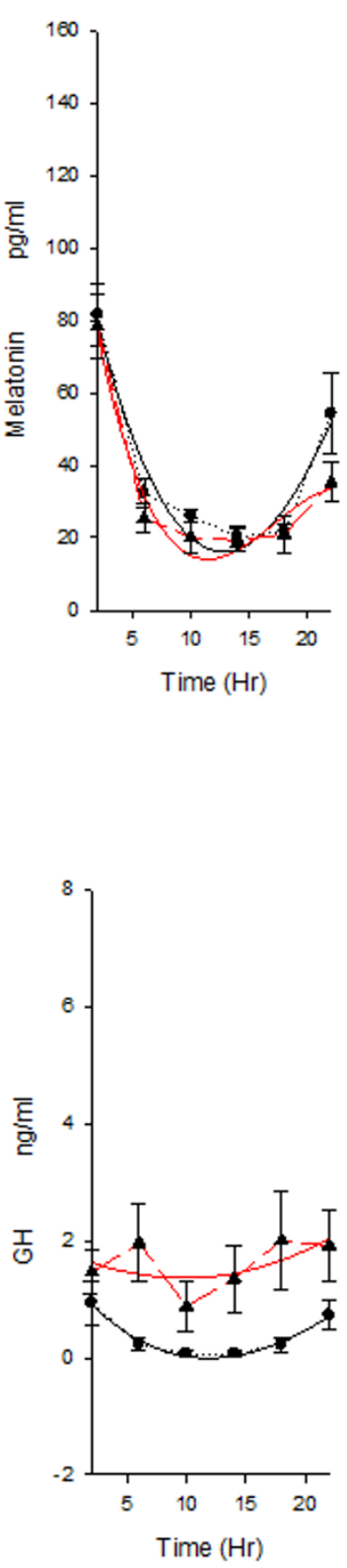
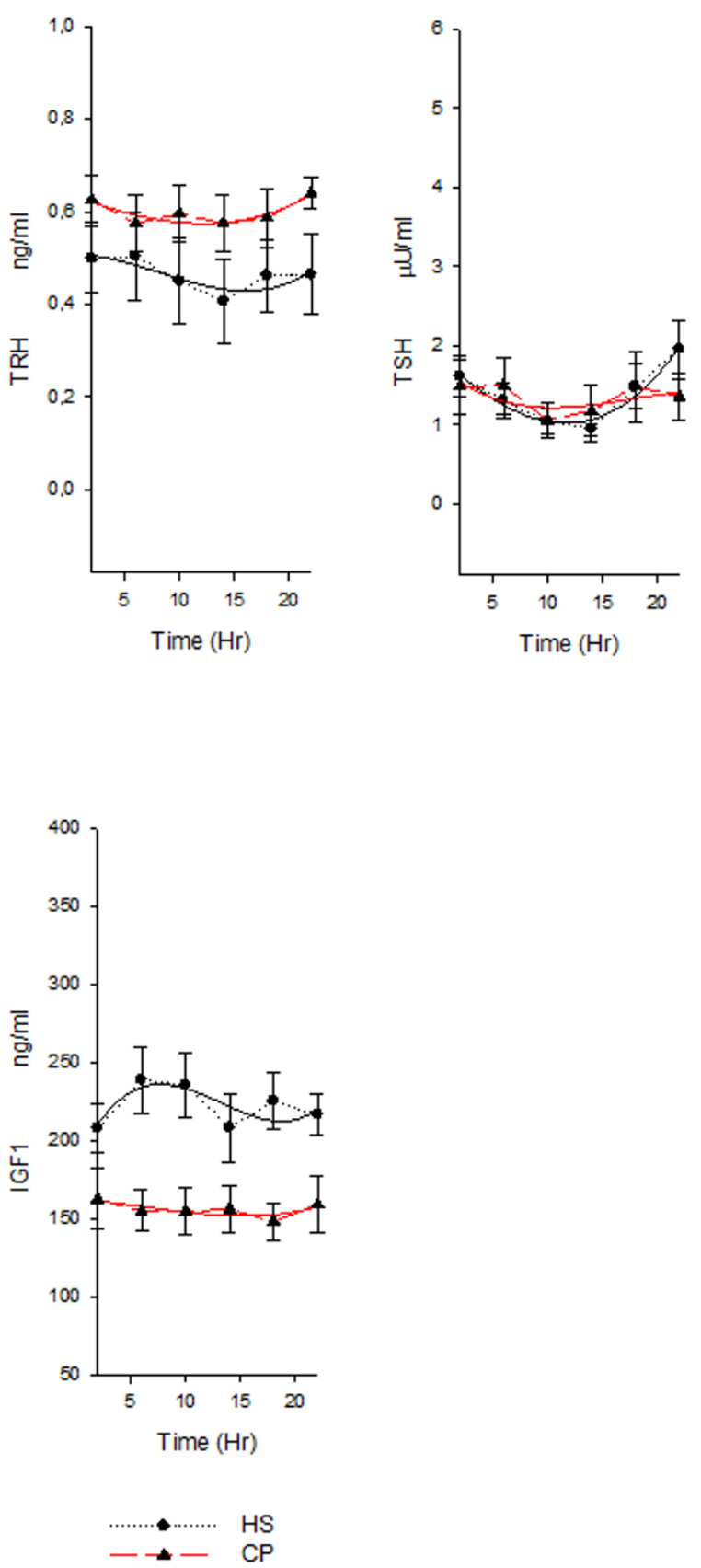

Figure 1 Nyctohemeral variation of hormone serum levels. $x$-y plot showing 24-hour time qualified profiles of cortisol, melatonin, TRH, TSH, free thyroxine, GH and IGF 1 expressed as mean \pm s.e. calculated on single time point values from ten healthy subjects and ten patients suffering from non small cell lung cancer. Cubic regression function data interpolation represented as best fit solid line superimposed on the raw data (dotted line).

(IL-2 and IL-4) $[13,14]$. The immunomodulatory role of melatonin may also be exerted by an influence on the thymic function mediated by TRH and TSH, able to counteract in experimental conditions thymic involution induced by prednisolone (this effect seems to be thyroid-independent and not correlated to thyroxine levels) [15].

TRH and TSH secretion changes with circadian periodicity and nightly peak hormone serum levels have been described. Thyroxine $\left(\mathrm{T}_{4}\right)$ and triiodothyronine $\left(\mathrm{T}_{3}\right)$ serum levels present minor changes during a 24-h period, their fluctuations are shortlived and diurnal variations in hormone levels can be demonstrated in most, but not in all instances. In our control subjects TRH and TSH blood levels show circadian variations with higher levels during the night, whereas thyroid hormone levels do not change with circadian rhythmicity. Melatonin may regulate the 

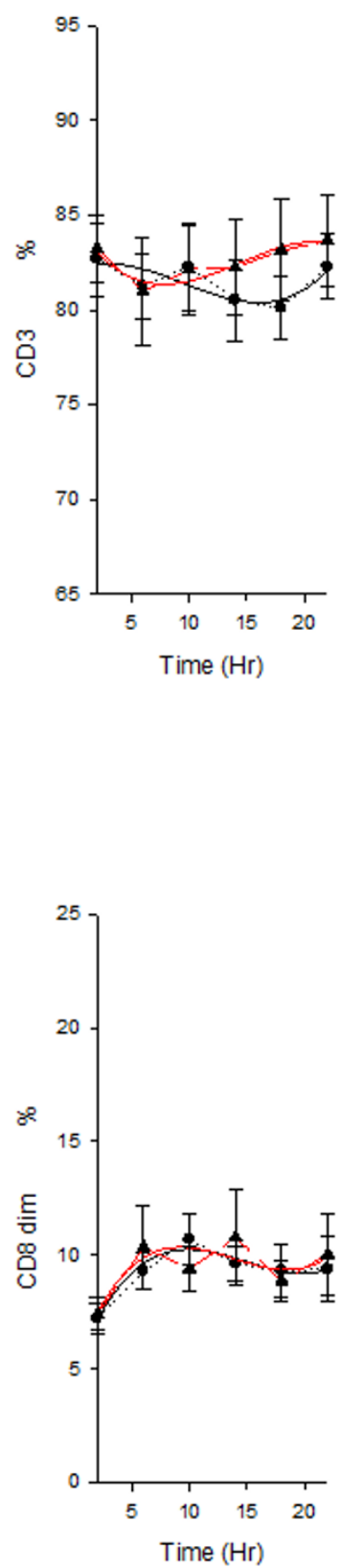
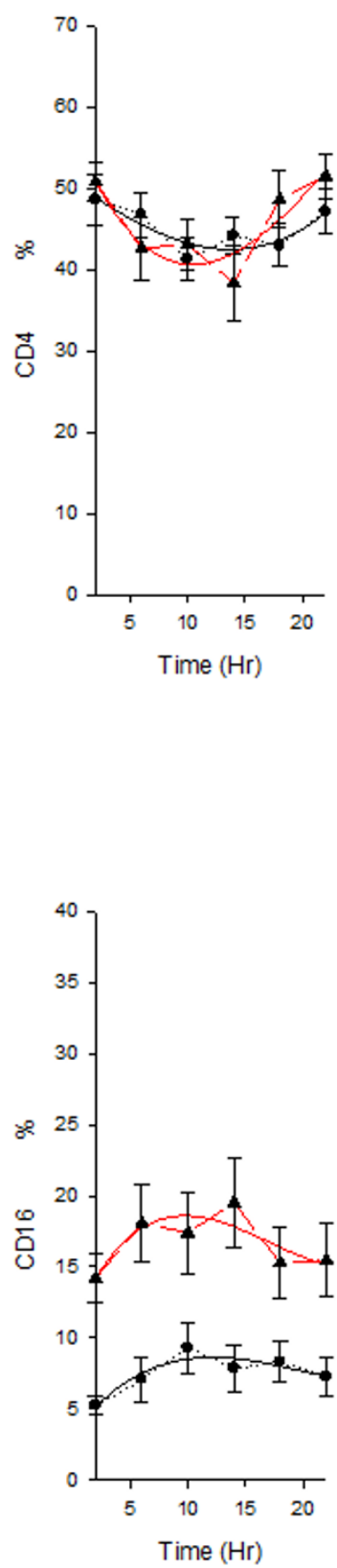
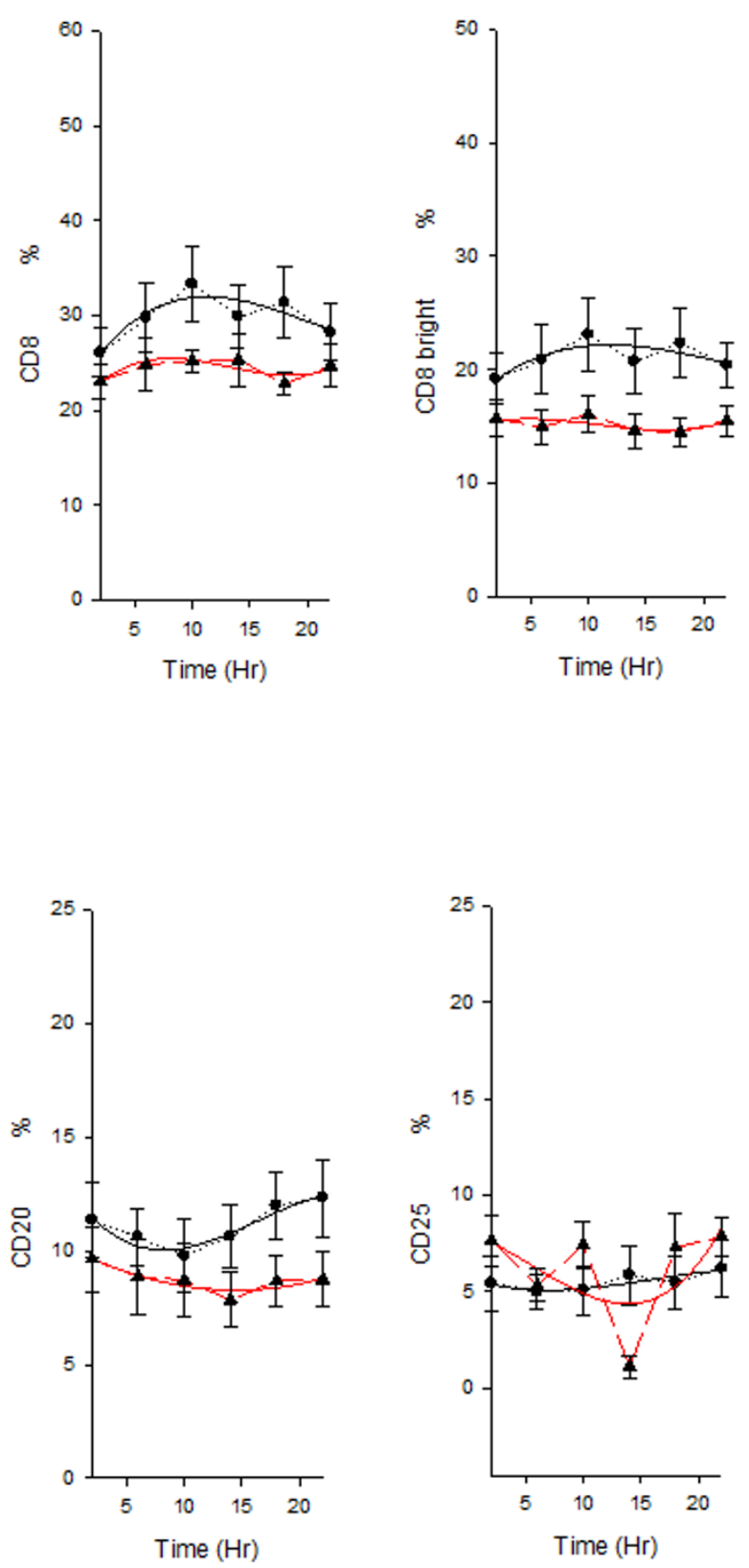

Figure 2 Nyctohemeral variation of lymphocyte subpopulations. $x$-y plot showing 24-hour time qualified profiles of lymphocyte subset percentages (CD3, CD4, CD8, CD8bright, CD8 dim, CD16, CD20, CD25) expressed as mean \pm s.e. calculated on single time point values from ten healthy subjects and ten patients suffering from non small cell lung cancer. Cubic regression function data interpolation represented as best fit solid line superimposed on the raw data (dotted line).

response of thyroid to hypothalamic-pituitary axis stimulation, playing a modulatory action on hypothalamicpituitary-thyroid axis and functioning as a servo-mechanism that diminishes or increases responses when stimuli are respectively too strong or too low $[16,17]$. This phenomenon, called feed-sideward, takes part also in the interaction among the pineal gland and the hypothalamic-pituitary-adrenal axis [18].
GH and IGF-1 have been recognized as stimulators of lymphopoiesis and immune function. IGF-1 is one of the most important growth factors for normal cell proliferation and several tumor cell lines have recently appeared to be also stimulated by this mitogen: it acts as an endocrine hormone via the blood and as paracrine and autocrine growth factor locally. $\mathrm{GH}$ stimulates the biosynthesis of IGF-1 in liver and in other organs and tis- 

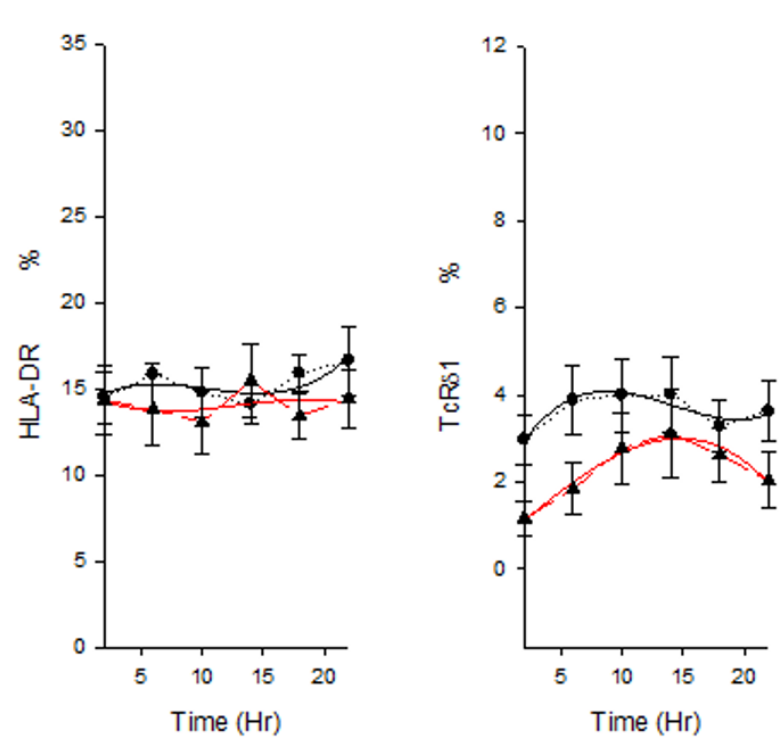
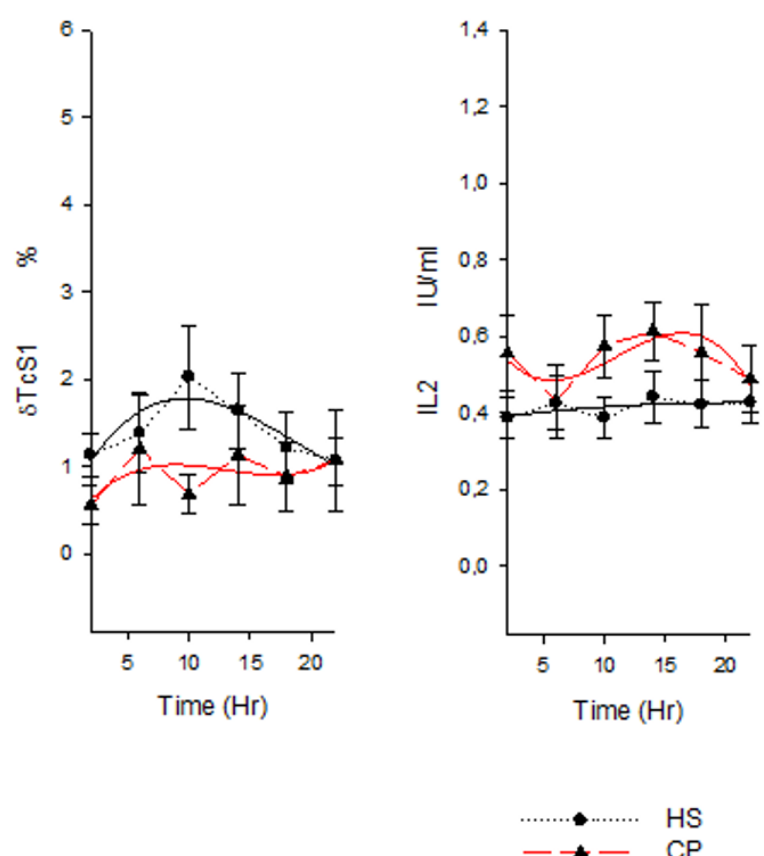

Figure 3 Nyctohemeral variation of lymphocyte subpopulations. $x$-y plot showing 24 -hour time qualified profiles of lymphocyte subset percentages (HLA-DR, TCR 1, $\delta T C S 1)$ and IL-2 serum levels expressed as mean \pm s.e. calculated on single time point values from ten healthy subjects and ten patients suffering from non small cell lung cancer. Cubic regression function data interpolation represented as best fit solid line superimposed on the raw data (dotted line).

sues and an autocrine or paracrine GH/IGF-1 system have been evidenced in lymphoid tissues, capable of influencing lymphopoiesis and immune function [19-21].

Neoplastic diseases are characterized by an altered pattern of hormonal secretion, with changes that may be qualitative (loss of circadian rhythmicity) and/or quantitative (increase or decrease of serum levels) as variously described by many scientific studies [22-25].

Immune responses show temporal variations, related to circadian changes of total lymphocytes and specific lymphocyte subsets in the peripheral blood, of antibodies and cell mediated immune responses, with an inverse relationship to plasma cortisol concentration [26-30]. Cortisol has a well recognized influence on immune function, inducing significant immunosuppression, characterized by the reduced cellular and humoral response of monocytes and $\mathrm{B}$ and $\mathrm{T}$ lymphocytes [31]. There are different circadian variations of the total number of circulating immune cells and of specific lymphocyte subpopulations [32,33]. As evidenced in our healthy control subjects, circulating $\mathrm{T}$ and $\mathrm{B}$ cell percentages levels change with circadian rhythmicity in antiphase with the rhythm of cortisol and this rhythm of variation is recognizable for the changes of total $\mathrm{T}$ cells, $\mathrm{T}$ helper/inducer subset, B cells and activated T cells, total B cells, T activated lymphocytes with expression of the $\alpha$ chain of IL-2 receptor. The levels of $\mathrm{T}$ suppressor/cytotoxic lymphocytes, natural killer cells and $\gamma \delta \mathrm{TCR}$ expressing cells are higher in the morning. Immune system plays an important role in the defense against neoplastic disease: there exists an effective T-cell mediated immune surveillance capable of monitoring the genetic integrity of mammalian cells, $\mathrm{T}$ lymphocytes are an essential component of specific immune responses wich produce tumour rejection and endocrine and immune alterations have been evidenced in cancer patients.

Data obtained in our study shows that in lung cancer patients cortisol, TRH, TSH and GH secretion is altered with loss of circadian rhythmicity. The disappearance of circadian rhythm of cortisol secretion is typical of hypercortisolism and adding to the reduced nocturnal melatonin secretion determines in our cancer patients the observed decrease of the melatonin/cortisol ratio. An altered pattern of cortisol secretion (absence of circadian rhythmicity and increased production), together with reduced nightly melatonin secretion, may represent a marker of neuro-endocrine system disorder [34]. The loss of circadian rhtyhmicity of cortisol serum level variation may be caused by an alteration of central nervous system (CNS) regulatory mechanisms of cortisol secretion and may be involved in a dual relationship to the melatonin secretion pattern [35]. The pineal gland influences the 
Table 1: Chronobiological data derived from best fitting sine curves (fitted period:24 hours $=360^{\circ}$ ) and $F$ and $p$ value from ANOVA performed between the timepoints for each variable

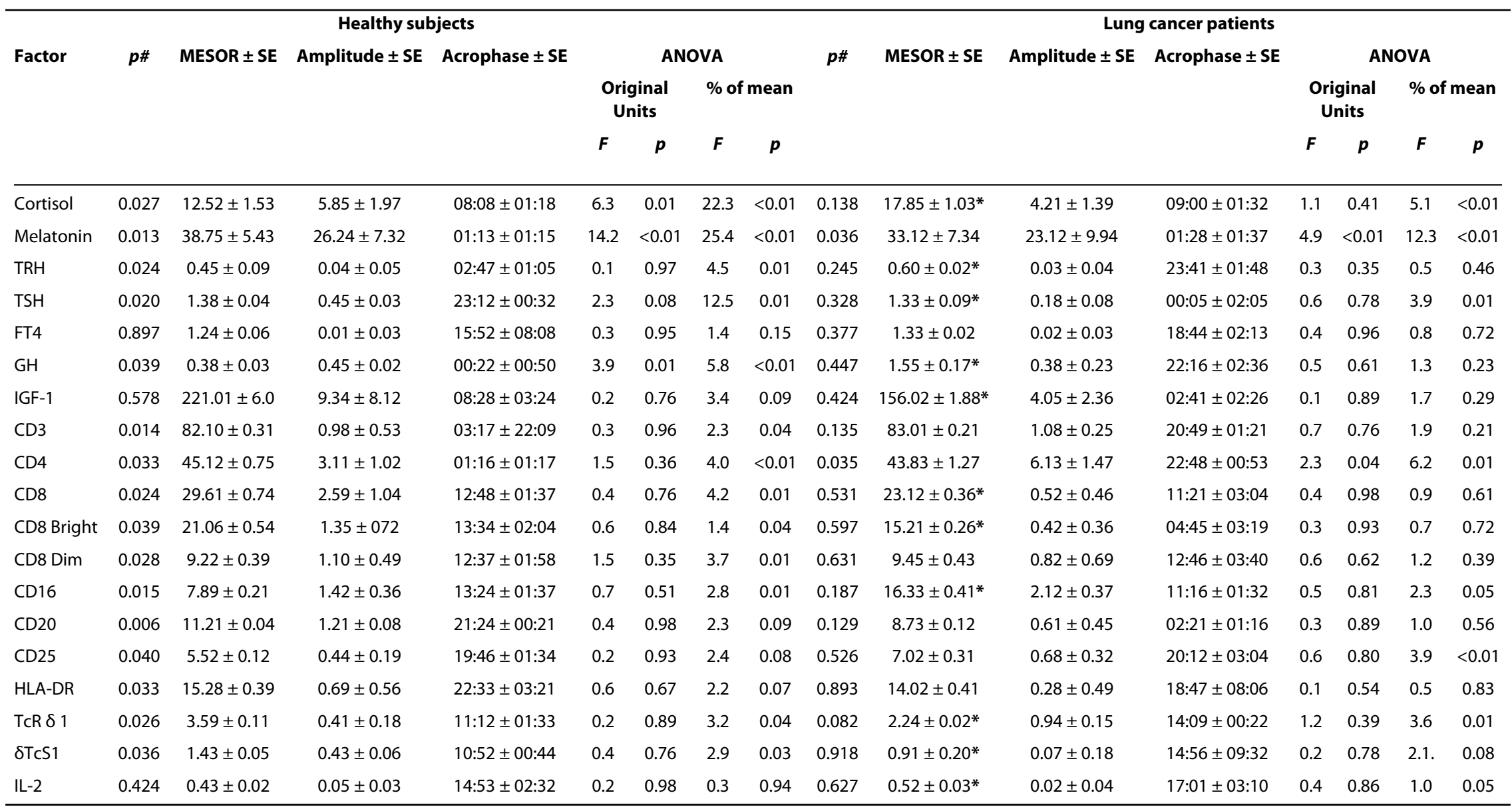

Units: $\mu \mathrm{g} / \mathrm{dl}$ for cortisol, $\mathrm{pg} / \mathrm{ml}$ for melatonin, $\mathrm{ng} / \mathrm{ml}$ for TRH, $\mu \mathrm{U} / \mathrm{ml}$ for TSH, $\mathrm{ng} / \mathrm{dl}$ for $\mathrm{FT}_{4}, \mathrm{ng} / \mathrm{ml}$ for GH, $\mathrm{ng} / \mathrm{ml}$ for IGF-l, percentage for lymphocyte subpopulations, IU/ml for IL-2; all parameters analyzed in all the subjects; $\mathrm{Hr}: \mathrm{Mn}$ for acrophase; all parameters analyzed in all the subjects. \# $p$ value from an $F$-test of the null amplitude rejection hypothesis (for a rhythm with a chosen period ?); $p$ value $<0.05$ from $t$-test or Mann-Whitney rank sum test as indicated performed on MESOR, amplitude and acrophase values 
function of the hypothalamus-hypophysis-adrenal axis, melatonin regulates glucocorticoid receptors and adrenal corticosteroids modulate pineal melatonin synthesis [36]. Data obtained in previous studies indicate that the pineal function is significantly impaired in patients with altered pattern of cortisol secretion or after the administration of dexamethasone $[37,38]$. The melatonin/cortisol ratio, calculated on the mean of nocturnal serum levels or on overnight urinary cortisol and melatonin (or its metabolite 6-hydroxy-melatonin-sulphate), may be a valuable and reliable method for population and individual screening for cancer at scheduled time intervals (for example, at yearly interval in the general population and at half-yearly interval or quarterly in subjects at elevated risk for neoplastic disease). There are reciprocal and different influences also among melatonin and male and female gonadal steroids and this is the reason why we have considered only male subjects to avoid sex related biases.

In our cancer patients $\mathrm{GH}$ serum levels are increased, but the nyctohemeral pattern of secretion is lost and this determines an unbalanced relationship between $\mathrm{GH}$ secretion and IGF-1 serum levels, that are decreased. Studies conducted on GH-deficient patients have demostrated that different time treatment schedules of $\mathrm{GH}$ administration have different effects on IGF-1 serum levels and the closest similarity to normal hormone and metabolite patterns and relationships is reached by $\mathrm{GH}$ injection in the evening [39]. All the patients enrolled in our study had normal BMI, so that the low levels of serum IGF-1 found in cancer patients should not be related to the state of nutrition. A global disorder of the neuroendocrine axes has to be considered and may also explain the increase of TRH levels and the decrease of TSH levels observed in our cancer patients. An important causal factor may be represented by the altered time structure of $\mathrm{GH}$ and $\mathrm{TRH}$ secretion evidenced in our lung cancer patients, but another explanation may be represented by hormone resistance. IL-2 serum levels are increased in our cancer patients, maybe following immune activation and maybe representative of a global increase of the others cyto/chemokines that mediate inflammation [40]. The changes in the TRH/TSH axis and GH/IGF-1 axis function in the patients suffering from cancer may be mediated by the inflammatory cytokines. Evidence from cell culture and animal experiments suggests that interleukin-1 (IL-1) and tumour necrosis factor- $\alpha$ (TNF $\alpha)$ can cause $\mathrm{GH}$ resistance. Increased circulating levels of the inflammatory cytokines and/or increased in vitro production by peripheral blood mononuclear cells have been reported in cancer patients. GH resistance with respect to the stimulation of hepatic IGF1 production has been observed in the lung cancer patients and GH resistance has been associated with an acute-phase response. The effects of the inflammatory cytokines are likely to be mediated by complex paracrine pathways, rather than 'endocrine' pathways [41,42].

The neuro-endocrine-immune system function is characterized by a complex time structure composed of multiple rhythms in different frequency ranges. The rhythms of the same frequency may have the same phase or different phases and usually show a well defined time-relation to each other. The loss of the array of rhythms or a change of their functional interactions may alter the organism's time structure leading to chronodisruption and internal desynchronization. The alteration of the organism's time structure may lead to functional disturbances and may impair repairing and defensive mechanisms. Cancer may arise from chronodisruption and may worsen internal desynchronization. A complete loss of rhythmicity or a change of phase of the rhythms are the most frequent alterations, but another important factor is represented by the change of levels and amplitude of variation.

The multifrequency structrure that characterizes the function of the immune system and the complexity of the time qualified variations of its different components has to be taken in consideration when we approach functional evaluations, clinical interpretations and therapeutical interventions. Among the lymphocyte subsets considered in our study, the CD8 bearing cells and in particular the $\mathrm{T}$ suppressor subset are diminished in lung cancer patients $[43,44]$. The decrease of $\mathrm{T}$ suppressor subpopulation has been observed in other types of cancer (gastric cancer, colorectal carcinoma and urological cancer) [45-47] and may represent a marker of immunological disorder. As evidenced in our study, the natural killer cells are increased in cancer patients, whereas the expression of the constant and variable domains of $\delta$ chain of Tcell receptor 1 is decreased and this may be an important finding as $\gamma \delta \mathrm{TCR}$ complex is mainly expressed at the cell surface of cytotoxic lymphocytes, is involved in $\mathrm{T}$ cell activation and activated $\gamma \delta$ expressing cells frequently exhibit cytotoxic activity against multiple target cell lines including neoplastic cells [48].

The altered time structure and serum level evidenced in our study for hypothalamic (TRH), pituitary $(\mathrm{GH}$, TSH), pineal (melatonin) and adrenal (cortisol) secretion may be produced by and/or may increase the altered interaction among nervous, endocrine and immune system. Lung cancer patients show loss of circadian rhythmicity of all lymphocyte subpopulations studied except for CD4. This phenomenon may impair an efficient immune response, may be related to altered hormone serum levels and to changes of the natural circadian rhythmicity and may cause the loss of the physiological timed windows of interaction among neural/endocrine structures and immune effectors, considering also that immune cells have hormone binding sites and hormone 
production capability. The reduction in number of CD8 and $\gamma \delta \mathrm{TCR}$ expressing cells in the peripheral blood may represent a marker of immunological disorder or may follow homing in pathological and neoplastic tissue as effector cells and/or regulatory cells.

In conclusion, our results have indicated that lung cancer is associated with disordered hormonal secretion and anomalies of proportion and circadian variation of lymphocyte subsets, probably expression of impaired integration among nervous, endocrine and immune system in front of advancing neoplastic disease. These systems show natural patterns of circadian rhythmicity and it is important to understand how multiple rhythms relate to each other to maintain an internal order and how the loss of this internal order may contribute to the beginning and the progression of neoplasticdisease.

\section{Competing interests}

'The authors declare that they have no competing interests'.

All the Authors have read and approved the submission of the present version of the manuscript and that the manuscript has not published and is not being considered for publication elsewhere in whole or in part in any language except as an abstract.

In the past five years we have not received reimbursements, fees, funding, or salary from an organization that may in any way gain or lose financially from the publication of this manuscript, either now or in the future. No organization is financing this manuscript (including the article-processing charge).

We do not hold any stocks or shares in an organization that may in any way gain or lose financially from the publication of this manuscript, either now or in the future

We Do no hold or are currently applying for any patents relating to the content of the manuscript. We have not received reimbursements, fees, funding, or salary from an organization that holds or has applied for patents relating to the content of the manuscript.

We have no other financial competing interests. There are no non-financial competing interests (political, personal, religious, ideological, academic, intellectual, commercial or any other) to declare in relation to this manuscript.

\section{Authors' contributions}

All authors have read and approved the final manuscript. GM: conception and design of the study, data collection, analysis and interpretation of data, carried out statistical analysis and the draft of the manuscript. GV: critical revisal of the manuscript. AD: interpretation of data, carried out part of the draft of the manuscript. SC: data collection, data interpretation, carried out part of the draft of the manuscript. RT: critical revisal of the manuscript, interpretation of data.

\section{Aknowledgments}

We are greatly indebted to Professor Franz Halberg and we wish to express our gratitude to him for introducing us to the study of Chronobiology

\section{Author Details}

'Unit of Internal Medicine and Chronobiology Unit, IRCCS Scientific Institute and Regional General Hospital, "Casa Sollievo della Sofferenza", Opera di Padre Pio da Pietrelcina S.Giovanni Rotondo (FG), Italy, ${ }^{2}$ Institute of Geriatrics, Department of Medical Science, University of Foggia, Italy and 3Department of Internal Medicine, University of Florence, Florence (FI), Italy

Received: 8 September 2009 Accepted: 21 June 2010

Published: 21 June 2010

\section{References}

1. Fauci AS: Mechanism of corticosteroid function on lymphocyte subpopulations.I. Redistribution of circulating $T$ and $B$ lymphocytes to the bone marrow. Immunology 1975, 28:669-80.

2. Reiter RJ: Functional pleiotropy of the neurohormone melatonin, antioxidant protection and neuroendocrine regulation. Front Neuroendocrinol 1995, 16:383-415.
3. Subramanian P, Mirunalini S, Dakshayani KB, Pandi-Perumal SR, Trakht I, Cardinali DP: Prevention by melatonin of hepatocarcinogenesis in rats injected with N-nitroso- diethylamine. J Pineal Res 2007, 43:305-12.

4. Subramanian P, Dakshayani KB, Pandi-Perumal SR, Trakht I, Cardinali DP: 24-hour rhythms in oxidative stress during hepatocarcinogenesis in rats: effect of melatonin or alpha-ketoglutarate. Redox Rep 2008, 13:78-86.

5. Cardinali DP, Cutrera RA, Esquifino Al: Psychoimmune neuroendocrine integrative mechanisms revisited. Bio/ Signals Recept 2000, 9(5):215-30.

6. Maestroni GJM, Conti A, Pierpaoli W: The pineal gland and the circadian. opiatergic, immunoregolatory role of melatonin. Ann N Y Acad Sci 1987, 496:67-77.

7. Lesnikov VA, Korneva EA, Dall'Ara A, Pierpaoli W: The involvement of pineal gland and melatonin in immunity and aging: II. Thyrotropinreleasing hormone and melatonin forestall involution and promote reconstitution of the thymus in anterior hypothalamic area (AHA)lesioned mice. Int J Neurosci 1992, 62:141-53.

8. Werner $\mathrm{H}$, LeRoith D: The role of the insulin-like growth factor system in human cancer. Adv Cancer Res 1996, 68:183-223.

9. Clark R: The somatogenic hormones and insulin-like growth factor-1: stimulators of lymphopoiesis and immune function. Endocrine Rev 1997, 18:157-179.

10. Lores Vazquez B, Pacheco Carracedo M, Oliver Morales J, Parada Gonzalez P, Gambon Deza F: Lymphocyte subpopulations of regional lymph nodes in human colon and gastric adenocarcinomas. Cancer Immunol Immunother 1996, 42:339-42.

11. Nelson W, Tong YL, Halberg F: Methods for cosinorrhytmometry. Chronobiologia 1979, 6:305-23.

12. Brzezinski A: Melatonin in humans. New Engl J Med 1997, 336:186-95.

13. Maestroni GJM: T-helper-2 lymphocytes as a peripheral target of melatonin. J Pineal Res 1995, 18:84-9.

14. Maestroni GJM, Conti A: The pineal nehurohormone melatonin stimulates activated CD4+, Thy-1+ cells to release opioid agonist(s) with immunoenhancing and anti-stress properties. J Neuroimmunol 1990, 28:167-176.

15. Pierpaoli W, Yi C: The involvement of pineal gland and melatonin in immunity and aging. I. Thymus-mediated, immunoreconstituting and antiviral activity of thyrotropin-releasing hormone. J Neuroimmunol 1990, 27:99-109.

16. Mazzoccoli G, Giuliani A, Carughi S, De Cata A, Puzzolante F, La Viola M, Urbano N, Perfetto F, Tarquini R: The hypothalamic-pituitary-thyroid axis and melatonin in humans: possible interactions in the control of body temperature. Neuroendocrinol Lett 2004, 25:368-72.

17. Vriend J: Evidence for pineal gland modulation of the neuroendocrinethyroid axis. Neuroendocrinol 1983, 36:68-78.

18. Sanchez de La Pena S: The feedsideward of cephalo-adrenal immune interactions. Chronobiologia 1993, 20:1-52.

19. Foekens JA, Portengen $H$, Janssen M, Klijn JG: Insulin-like growth factor-1 receptors and insulin-like growth factor-1 like activity in human primary breast cancer. Cancer 1989, 63:2139-2147.

20. Foekens JA, Portengen $H$, van Putten WL, Trapman AM, Reubi JC, AlexievaFigusch J, Klijn JG: Prognostic value of receptors for insulin-like growth factor 1, somatostatin, and epidermal growth factor in human breast cancer. Cancer Res 1989, 15:7002-7009.

21. Auernhammer CJ, Strasburger CJ: Effects of growth hormone and insulin-like growth factor on the immune system. Eur J Endocrinol 1995, 133:635-645.

22. Mazzoccoli G, Giuliani A, Bianco G, De Cata A, Balzanelli M, Carella AM, La Viola M, Tarquini R: Decreased serum levels of insulin-like growth factor (IGF)-I in patients with lung cancer: temporal relationship with growth hormone (GH) levels. Anticancer Res 1999, 19:1397-9.

23. Bartsch C, Bartsch H, Schmidt A: Melatonin and 6-sulfatoxymelatonin circadian rhythms in serum and urine of primary prostate cancer patients, evidence for reduced pineal activity and relevance of urinary determinations. Clin Chim Acta 1992, 209:153-167.

24. Bartsch C, Bartsch H, Karenovics A: Nocturnal urinary 6sulphatoxymelatonin excretion is decreased in primary breast cancer patients compared to age-matched controls and shows negative correlation with tumor-size. J Pineal Res 1997, 23:53-8.

25. Mazzoccoli G, Carughi S, De Cata A, La Viola M, Giuliani A, Tarquini R, Perfetto F: Neuroendocrine alterations in lung cancer patients. Neuroendocrinol Lett 2003, 24:77-82. 
26. Ratte J, Halberg F, Kuhl JFW: Circadian variation in the rejection of rat kidney allografts. Surgery 1973, 73:102-8.

27. Knapp MS, Cove Smith JR, Dugdale R: Possible effect of time on renal allograft rejection. BMJ 1979, 1:75-7.

28. Calderon RA, Thomas DB: In vivo cyclic change in B-lymphocyte susceptibility to T-cell control. Nature 1980, 285:662-664.

29. Abo T, Kumagi K: Surface studies of immunoglobulins on human B lymphocytes. III. Physiological variations of Slg+ cells in peripheral blood. Clin Exp Immunol 1978, 33:441-52.

30. Abo T, Kawate T, Itoh K: Characterization of rhythms and cell components in circadian variation of human and mouse lympocytes. Chronobiologia 1979, 6:71-2.

31. Maestroni GJ, Conti A, Pierpaoli W: Role of the pineal gland in immunity. Circadian synthesis and release of melatonin modulates the antibody response and antagonizes the immunosuppressive effect of corticosterone. J Neuroimmunol 1986, 13:19-30.

32. Mazzoccoli G, Correra M, Bianco G, De Cata A, Balzanelli M, Giuliani A, Tarquini R: Age-related changes of neuro-endocrine-immune interactions in healthy humans. J Biol Regul Homeost Agents 1997, 11:143-7.

33. Mazzoccoli G, Bianco G, Correra M, Carella AM, Balzanelli M, Giuliani A, Tarquini R: Circadian variation of lymphocyte subsets in healthy subjects. Rec Prog Med 1998, 89:569-72.

34. Piovesan A, Terzolo M, Borretta G: Circadian profile of serum melatonin in Cushing's disease and acromegaly. Chronobiol Int 1990, 7:259-261.

35. Persengiev SP: Multiple domains of melatonin receptor are involved in the regulation of glucocorticoid receptor-induced gene expression. $J$ Steroid Biochem Mol Biol 1999, 68:181-7.

36. Sainz RM, Mayo JC, Reiter RJ: Melatonin regulates glucocorticoid receptor: an answer to its antiapoptotic action in thymus. FASEB-J 1999, 13:1547-56

37. Demish L, Demish L, Nickelsen T: Inflluence of dexamethasone on nocturnal melatonin production in healthy adult subjects. $J$ Pineal Res 1988, 5:317-21.

38. Mazzoccoli G, Carughi S, De Cata A, La Viola M, Vendemiale G: Melatonin and cortisol serum levels in lung cancer patients at different stages of disease. Med SciMonit 2005, 11:CR284-288.

39. Blum WF, Albertsson-Wikland K, Rosberg S, Ranke MB: Serum levels of insulin-like growth factor I (IGF-I) and IGF binding protein 3 reflect spontaneous growth hormone secretion. J Clin Endocrinol Metab 1993, 76:1610-1616.

40. Orditura M, Romano C, De Vita F: Behaviour of interleukin-2 serum levels in advanced non-small-cell lung cancer patients: relationship with response to therapy and survival. Cancer Immunol Immunother 2000 49:530-6.

41. von Laue S, Ross RJ: Inflammatory cytokines and acquired growth hormone resistance. Growth Horm IGF Res 2000, 10:S9-14.

42. Shumate ML, Yumet G, Ahmed TA, Cooney RN: Interleukin-1 inhibits the induction of insulin-like growth factor-l by growth hormone in CWSV-1 hepatocytes. Am J Physiol Gastrointest Liver Physiol 2005, 289:G227-39.

43. Mazzoccoli G, Grilli M, Carughi S, Puzzolante F, De Cata A, La Viola M, Giuliani A, Urbano N, Tarquini R, Perfetto F: Immune system alterations in lung cancer patients. Int J Immunopathol Pharmacol 2003, 16:167-74.

44. Mazzoccoli G, Balzanelli M, Giuliani A, De Cata A, La Viola M, Carella AM, Bianco G, Tarquini R: Lymphocyte subpopulations anomalies in lung cancer patients and relationship to the stage of disease. In Vivo 1999, 13:205-9.

45. Kaver I, Pecht M, Trainin N, Greenstein A, Braf Z: T lymphocyte subsets and function in the peripheral blood of patients with urological cancer. Oncology 1992, 49:108-13.

46. Takahashi M, Fujimoto S, Takai M, Ohno K, Endoh F, Masuda Y, Obata G: Two-color flow cytometric analysis of splenic lymphocyte subpopulations in patients with gastric cancer. Surg Today 1992, 22:35-9.

47. Jackson PA, Green MA, Marks CG, King RJ, Hubbard R, Cook MG: Lymphocyte subset infiltration patterns and HLA antigen status in colorectal carcinomas and adenomas. Gut 1996, 38:85-9.

48. Kabelitz D, Wesch D, He W: Perspectives of gammadelta T cells in tumor immunology. Cancer Res 2007, 67:5-8.

Pre-publication history

The pre-publication history for this paper can be accessed here:

http://www.biomedcentral.com/1471-2407/10/314/prepub doi: 10.1186/1471-2407-10-314

Cite this article as: Mazzoccoli et al., Altered time structure of neuro-endocrine-immune system function in lung cancer patients BMC Cancer 2010 10:314

\section{Submit your next manuscript to BioMed Central} and take full advantage of:

- Convenient online submission

- Thorough peer review

- No space constraints or color figure charges

- Immediate publication on acceptance

- Inclusion in PubMed, CAS, Scopus and Google Scholar

- Research which is freely available for redistribution 